\title{
Factores que Influyen en el Aprendizaje del Idioma Inglés de Nivel Inicial en una Universidad Chilena
}

\author{
María J. Valenzuela ${ }^{(1)}$, Karen Romero ${ }^{(2)}$, Cristian Vidal-Silva ${ }^{(3)}$ y Andrew Philominraj ${ }^{(4)}$ \\ (1) Servicio de Salud Maule, 1 Sur 546, Talca-Chile (e-mail: mjvalenzuela@ssmaule.cl) \\ (2) Colegio Concepción, 12 1 1/2 Sur C, 6 Poniente S/N, Talca-Chile (e-mail: karenromerog@gmail.com) \\ (3) Dpto. de Ingeniería en Informática, Facultad de Ingeniería, Univ. Autónoma de Chile, 5 Poniente 1670, \\ Talca-Chile (e-mail: cristian.vidal@uautonoma.cl) \\ (4) Programa de Idiomas, Facultad de Ciencias de la Educación, Univ. de Talca, Av. Lircay s/n, Talca-Chile \\ (e-mail: andrew@utalca.cl)
}

Recibido May. 11, 2016; Aceptado Jul. 6, 2016; Versión final Ago. 15, 2016, Publicado Dic. 2016

\begin{abstract}
Resumen
El objetivo de este estudio es conocer el efecto que tienen el profesor, las herramientas de apoyo y la dependencia educacional de procedencia en el aprendizaje del inglés. El impacto se midió a través de un estudio descriptivo-correlacional, utilizando el Oxford Placement Online Test como pre y post a una muestra de 132 estudiantes de nivel inicial del idioma inglés de diversas carreras de la Universidad de Talca en Chile. Los estudiantes fueron divididos en cursos con tres herramientas de apoyo diferentes entre medición. Los resultados indican que no hay diferencias significativas entre las herramientas de apoyo utilizadas en el rendimiento de los estudiantes. Tampoco se observa influencia al analizar la dependencia educacional de procedencia, siendo el profesor el único factor que resulta relevante en este proceso. De esta forma se puede concluir que a pesar del desarrollo de sistemas computacionales para el aprendizaje, el profesor sigue siendo un factor determinante dentro de este ámbito.
\end{abstract}

Palabras clave: aprendizaje del inglés, segundo idioma, herramientas de apoyo, profesor, dependencia educacional, educación superior

\section{Factors that Influence the Learning of English by Freshman Students in a Chilean University}

\begin{abstract}
The aim of this study was to know the effects of the teacher, support tools and educational dependency on the process of teaching and learning of English. The impact was measured through a correlational descriptive study using the Oxford Placement Test as pre- and post-testing tool administered to a sample of 132 beginner level of English students, all belonging to different careers at the University of Talca in Chile. The results show no significant difference in the use of support tools or educational dependency in students' performance. It is the teacher who emerges as the only relevant factor in this process. Thus, it can be concluded that despite the development of computer systems for learning, the teacher continues to remain a determining factor in this area.
\end{abstract}

Keywords: learning English; second language; support tools; teacher; educational dependency; higher education 


\section{INTRODUCCIÓN}

El aprendizaje de nuevos idiomas es un tema que lleva en la palestra más de dos mil años. En la actualidad, existe una evolución de metodologías y herramientas en la búsqueda de una estrategia efectiva o universal para enseñar el idioma inglés. Estos estudios han convocado a pedagogos, lingüistas y psicólogos, lo que ha generado un alto desarrollo metodológico (Segura, 2012; Thanasoulas, 2015), paralelamente, acompañado por avances tecnológicos, que hoy en día tienen un rol protagónico en la dinámica de enseñanza-aprendizaje y dictan la pauta de cómo se enseña idioma Inglés en el mundo. Se ha establecido que a pesar de que los textos y las horas de conversación desde hace tiempo son parte de la enseñanza de inglés, hoy en día el desarrollo tecnológico ha permitido que surjan herramientas basadas en programas computacionales, muy prometedoras, ya que reúnen los elementos de gramática y oralidad que se persiguen para el aprendizaje del inglés (Emerging Tools, 2016; Herramientas, 2016).

Los últimos años se han caracterizado por un fenómeno social identificado como el "boom" del uso de las tecnologías de información y comunicación (TICs), los desarrollos del multimedia y la accesibilidad masiva al internet le han dado a las computadoras un rol dentro de la enseñanza de idiomas, que ha significado tanto en profesores como en alumnos una actualización de las herramientas didácticas tradicionales (Moreno, 2011). Justamente, el Inglés ha sido uno de los pioneros en la utilización de la tecnología en su enseñanza desarrollándose sistemas tutoriales propios llamados Sistemas para Aprendizaje de Lenguas Asistido por Computadores Inteligentes (CALL) (Duran y Barrios, 2007; Blake, 2015; Herramientas, 2016). Los autores de este trabajo afirman que, tanto para alumnos como para profesores, existen ventajas y utilidades de CALL por sobre otras herramientas: (1) permite que el profesor asuma un papel de facilitador, que explore nuevas vías y métodos de enseñanza, y que destine tiempo a otras actividades pedagógicas (Moreno, 2011). (2) Se logra aumentar la motivación de los estudiantes, ayudando a que asuman un papel más activo en su aprendizaje. Además, tal y como resalta Blake (2015) CALL facilita el aprendizaje con distintas estrategias y estilos, y promueve el aprendizaje colaborativo (Ramírez y Alonzo, 2007), y su efectividad es alta dado el actual uso de tecnologías.

El aprendizaje combinado, enfatiza el rol que las TICs cumplen e indican y que es posible crear un aprendizaje en los estudiantes más "distribuido" (Graham, 2006; Simasathiansophon, 2014), dada una mayor variedad de recursos que permiten obtener conocimiento sin necesidad de estar dentro del salón de clases, ni con un profesor que indique contenidos a estudiar y aprender (Pérez et al., 2014). Además, se promueve el contacto entre docente y estudiante y entre los mismos pares a través de los recursos que entrega la tecnología, para así, no sólo promover el aprendizaje mediante una supervisión directa, sino también mediante el trabajo autónomo del alumno (Simasathiansophon, 2014). Claramente, las herramientas para la enseñanza de inglés han evolucionado para tener un formato que intenta emular el comportamiento del docente, para que el estudiante aprenda de forma autónoma, recibiendo incluso correcciones automáticas. Sin embargo, no se ha llegado a un consenso que defina cuál de todas las herramientas disponibles garantizaría un aprendizaje efectivo en Chile y en el mundo, aun cuando diversos estudios proponen al método combinado como el que presenta mejores resultados en contextos de habla hispana (Pérez et al., 2014; Silva, 2015).

En vista de la importancia del Inglés en términos de relaciones internacionales y producción científica, se hace latente la necesidad de conocer la dinámica que guía la enseñanza de este idioma, cuyo foco de instrucción ha cambiado su enseñanza como desde un código formal hasta un medio para lograr comunicación (Segura, 2012). Así mismo, la dinámica del aula se ha visto intervenida debido a la inclusión de las TICs como herramientas de apoyo, junto con un cambio de rol en profesores y alumnos. Por tanto, mientras el profesor antes tenía un rol directivo en el aula, ahora es un ente facilitador de conocimientos que guía al estudiante al desarrollo de competencias (Martínez, 2007), mientras los alumnos ahora asumen un rol protagónico en su formación académica, gestionando por si mismos su propio conocimiento (Piquer, 2006).

\section{ENSEÑANZA DEL INGLÉS}

El proceso de enseñanza - aprendizaje del Inglés como segundo idioma será analizado desde un modelo educativo con enfoque combinado, que, a diferencia de un enfoque tradicional, enfatiza el rol que tiene el profesor, el alumno y las TICs dentro de este proceso, siendo estas últimas las que entregan una mayor variedad de recursos que permiten obtener conocimiento (Constantinou y Zacharia, 2003). A continuación se describen cada uno de los componentes o factores antes mencionados:

1. Un profesor según el enfoque tradicional es el responsable de intencionar la construcción de conocimiento en los estudiantes, teniendo un rol directivo en el aula. Por lo tanto, ningún cambio efectivo se producirá sin el apoyo y compromiso del profesor, ya que el desarrollo de la educación depende de lo que los profesores decidan y de sus acciones para con este proceso (Pérez et al., 2014). Dados los cambios en 
el foco de atención para la enseñanza del inglés, se espera que el profesor se adecúe a estos cambios (Panteeva, 2015).

Las nuevas líneas en la enseñanza de Inglés apuntan a modelos de enseñanza-aprendizaje más abiertos, enfocados en el individuo y cómo este aprende (Brutt-Griffler, 2002). Gracias a esto, tal y como señala Segura (2012), se han desarrollado transformaciones cualitativas en la práctica en aulas, que hacen necesario focalizar la atención en el estudio y la comprensión del proceso de aprendizaje tomando en cuenta factores relativos a la personalidad del estudiante.

2. Un alumno también se debe adaptar a las nuevas tendencias de educación, teniendo cada día un rol más activo en su propio aprendizaje para gestionar el desarrollo de su conocimiento (Piquer, 2006). Los alumnos han debido gestionar, además, la utilización de estrategias adecuadas en el proceso de enseñanzaaprendizaje de idioma Inglés, con una mayor motivación por aprender (Tuncer, 2009; Derakhshan et al., 2015).

3. Otro de los componentes con un factor de ayuda a lograr un entendimiento global del aprendizaje del idioma Inglés, son las herramientas de apoyo, las que cobran importancia para guiar los contenidos de la clase, ya que, tal y como señalan (Batista y Salazar, 2003; Vergara y Alegría, 2010), es habitual que un profesor adapte su clase a los lineamientos que las herramientas entregan. Claramente, la elección de herramientas adecuadas debe ser en función de los objetivos de aprendizaje y competencias ya definidas (Derakhshan et al., 2015). Los contenidos y herramientas de evaluación de estos instrumentos ayudan a los estudiantes a desarrollar cuatro habilidades que representan el objetivo de la adquisición del inglés como segundo idioma: hablar, comprender el idioma oral, leer y escribir (Valdés, et. al., 2015). Entre las herramientas utilizadas para la enseñanza y aprendizaje de idioma Inglés, se destacan:

(i) Los libros de texto de acuerdo a las necesidades de aprendizaje y herramientas prácticas de oralidad, tradicionalmente clases de conversación y repetición simultánea de conceptos, formas básicas de interacción lingüística (Unamuno, 2003; Vergara y Alegría, 2010); por lo cual, resulta fundamental el desarrollo de relaciones comunicativas en el aula. Los avances en la tecnología han permitido a los diseñadores de textos de estudio, seleccionar herramientas de apoyo didáctico genuinas para el desarrollo de la oralidad y la escritura (AI-Seghayer, 2001; Laufer y Hill, 2000). En este contexto, actualmente los libros cuentan con sesiones dentro del contenido, que dan espacio para la conversación del tema tratado, a fin de acelerar y consolidar las habilidades comunicativas de los alumnos (Carroli, 2001).

(ii) Modalidades de software educativos, cuya clasificación es determinada por la tarea que desempeñan y la etapa del proceso de enseñanza a la que se enfocan (González, 2002; Herramientas, 2016): Tutoriales, Hipermedia, Ejercitadores, Simulaciones, Juegos, Herramientas de Aprendizaje, y Evaluaciones. Es importante destacar que todos los software educativos para el aprendizaje de una idioma extranjero se diferencian en el papel que cumplen dentro de este proceso, esto es, mientras unos pretenden emular el comportamiento del docente como guía para el aprendizaje, otros buscan la ejercitación sistemática de lo que se desea enseñar (Pressman, 2010; Sawaki, 2001). A través de estos software, es posible analizar y entender datos reales y cotidianas de un idioma, por ejemplo el lenguaje coloquial de los intercambios de bienes y de los servicios en tiendas y restaurantes (Subrahmanyam et al., 2013; Zou y Li, 2015).

Las tecnologías al servicio de la enseñanza del Inglés son vistas útiles para el aprendizaje y la investigación de idiomas, ya que los estudiantes pueden leer los textos en línea con una gran variedad de asistentes de aprendizaje tales como traductores y diccionarios con audio, además de interacciones de voz y auditivas (Simasathiansophon, 2014; Herramientas, 2016). Claramente, las herramientas para la enseñanza de Inglés han evolucionado lo suficiente para tener un formato que intente emular el comportamiento del docente, brindando mayor autonomía en la forma en la que el estudiante aprende, recibiendo incluso correcciones automáticas (Batista y Salazar, 2003; Simasathiansophon, 2014). Según Moreno (2011), estos recursos tecnológicos no reemplazan al profesor, pero si son un poderoso aliado en el proceso de enseñanza y aprendizaje. Además, no existe un consenso respecto a cuales herramientas garantizan un aprendizaje efectivo. Atendiendo a esto, es que surge el objetivo de conocer el efecto que tienen el profesor y las herramientas de apoyo en el proceso de enseñanza-aprendizaje del idioma Inglés, así como también, determinar si la dependencia educacional predice un mejor nivel del dominio del idioma.

\section{ESTUDIOS ESTADÍSTICOS}

Para evaluar la efectividad de cada uno de los factores, se utiliza el instrumento Oxford Placement Online Test (OPOT, 2016); prueba que adapta la dificultad de las preguntas en función del rendimiento del estudiante. Por ejemplo, si el estudiante falla en su respuesta, el programa baja la dificultad de la siguiente pregunta. Como esta prueba es adaptativa, el número de preguntas fluctúa entre 40 a 45 , y su tiempo de 
aplicación es de 40 a 45 minutos. Dentro de los resultados que arroja esta prueba, se encuentran los puntajes brutos, junto con su conversión al nivel de dominio del estudiante, de acuerdo a la estandarización que propone la CEF del Marco Común Europeo (Consejo de Europa, 2002), la misma que utiliza el programa de idiomas de la Universidad de Talca. Además, es relevante destacar que este instrumento es validado a nivel internacional (OET, 2016).

La aplicación del Oxford Placement Online Test es de forma individual, mediante un computador con conexión a Internet que accede al sistema a través de un e-mail y una contraseña que entregan los evaluadores. La prueba consta de 5 partes donde cada una equivale al $20 \%$ del total de la prueba: 1 . Reading: habilidad y comprensión lectora del estudiante, (34 preguntas aproximadamente); 2. Writing: capacidad de escritura del alumno, (40 puntos); 3. Use of English: facultad para hacer buen uso del vocabulario y la gramática al construir oraciones, (50 preguntas aproximadamente); 4. Listening: comprensión de un discurso hablado en Inglés (30 preguntas); y 5. Speaking: contestar preguntas en una conversación en inglés (40 puntos).

\section{Descripción de la muestra}

La muestra es no probabilística la que se establece por conveniencia ya que se utilizaron los datos de 132 alumnos pertenecientes a diversas carreras de pre grado que terminaron el curso Beginner English 1 (curso inicial de inglés en el programa de idiomas de Universidad de Talca) y que se evaluaron al final del proceso con el mismo Test del inicio (Oxford Placement Online Test). El 53,8\% de los estudiantes pertenecen al campus Lircay y el $46,2 \%$ al campus Los Niches de la Universidad de Talca. Sus edades fluctúan entre los 18 y 29 años de edad (edad promedio $\bar{X}=20,57$; desviación estándar edad $\sigma=2,71$ ), siendo un $59,1 \%$ hombres y un $40,9 \%$ mujeres. En lo relativo al tipo de establecimiento del que los estudiantes egresan de enseñanza media, se observa que el $12,1 \%$ pertenece a colegios particulares pagados, el $43,9 \%$ a particulares subvencionados y un 43,9\% a municipales. Los profesores que participaron en este estudio fueron 4: Profesor A, Profesor B, Profesor C y Profesor D; donde el profesor A estuvo a cargo de 37 estudiantes, el profesor B de 24 estudiantes, el profesor $\mathrm{C}$ de 37 estudiantes y el Profesor $\mathrm{D}$ de 34 alumnos, respectivamente. Según la herramienta de apoyo utilizada, 50 alumnos utilizaron Dyned, 38 Interchange y 44 Top Notch.

\section{Procedimiento}

La logística, coordinación y acceso a los datos utilizados para la realización de este estudio fueron aportados por el Programa de Inglés de la Universidad de Talca. Para llevar a cabo este estudio, en primer lugar, se establecieron tres metodologías combinadas, donde la única diferencia entre ellas es la herramienta de apoyo utilizada en la clase. Sus contenidos fueron homologados con el objetivo de enseñar lo mismo en los tres tratamientos (ver Tabla 1).

Tabla 1: Herramientas de estudio de aprendizaje de idioma Inglés.

\begin{tabular}{|c|c|c|c|}
\hline Interchange & Texto Interchange & CD-Rom del texto & Conversación \\
\hline Top Notch & Texto Top Notch & CD-Rom del texto & Conversación \\
\hline DynEd & Software DynEd & guías de apoyo & Conversación \\
\hline
\end{tabular}

Tal y como se detalla en la Tabla 2, cada profesor trabajó con tres secciones, cada una de las cuales tenía un número aproximado de 20 estudiantes de carreras afines. Cada una de estas secciones es guiada con una herramienta diferente, las que se detallan a continuación:

1. Libro de texto Interchange $3^{\text {ra }}$ Edición: Libro de texto diseñado para el aprendizaje del idioma inglés. Se compone de un texto para el profesor y otro para el alumno que incluye un CD-Rom de audio con ejercicios de auto-aprendizaje (Richards et al., 2005).

2. Texto Top Notch: Libro de Texto con ediciones para estudiante y profesor con CD-Rom con actividades para imprimir y una prueba de colocación completa del curso (profesor), mientras la edición para el estudiante contiene ejercicios para la clase, ejercicios para el hogar y un video de programa de televisión (Saslow et al., 2003).

3. DynEd (Educación Dinámica): Software que cubre niveles de competencia en el idioma y está diseñado, con cursos acordes a las distintas etapas del ciclo vital. Posee ejercicios de voz y proporciona retroalimentación constructiva para profesores y estudiantes a través de una tutoría. Además, cuenta con un avanzado sistema de registros que ofrece un seguimiento en línea del progreso del estudiante. La herramienta DynEd, permite desarrollar la capacidad de autonomía del estudiante (Dyned, 2014). 
Tabla 2: Distribución del profesor por sección y herramientas de apoyo.

\begin{tabular}{|c|l|l|l|}
\hline Profesor & DynEd & Top Notch & Interchange \\
\hline$A$ & Sección 12 & Sección 10 & Sección 9 \\
\hline$B$ & Sección 11 & Sección 7 & Sección 8 \\
\hline$C$ & Sección 4 & Sección 3 & Sección 5 \\
\hline$D$ & Sección 6 & Sección 1 & Sección 2 \\
\hline
\end{tabular}

Designadas las secciones y las herramientas a utilizar por cada profesor, durante las primeras clases, se llevó a cabo el Oxford Placement Test Online (pre-test). Luego de eso, las clases se desarrollaron de forma normal siguiendo las pautas de la herramienta asignada y realizando una evaluación al final del curso con el mismo instrumento que se aplicó al inicio (post-test).

\section{RESULTADOS}

La distribución de los puntajes brutos de los estudiantes en los pre y post-test presentan un ajuste normal de sus datos, de acuerdo a lo obtenido mediante el análisis con la prueba Kolmogorov-Smirnov (Sheskin, 2003), cuya significación bilateral en el caso del pre-test fue de un 0,421 y en el post-test de un 0,266, lo que indica que ambas muestras tienen una distribución que se ajusta a la curva normal.

Conocida la distribución normal de los datos, se utiliza la prueba t de Student (Sheskin, 2003) con el fin de realizar una comparación de medias para muestras relacionadas. La aplicación de esta prueba no presenta diferencias estadísticamente significativas entre las medias de los niveles de dominio del idioma, arrojados por el pre y post-test de los estudiantes: distribución $t=-0,957$; grados de libertad gl $=131 ;$ valor $p=0,340$. Lo que indica que entre la primera y segunda aplicación, los estudiantes no avanzaron al siguiente nivel de dominio del idioma desde A1 a A2. Sin embargo, al analizar la diferencia de medias entre el pre y post-test de los puntajes brutos obtenidos, se encuentran diferencias estadísticamente significativas, lo que evidencia un avance en el aprendizaje de los estudiantes, pero no lo suficiente como para pasar al siguiente nivel en el dominio del idioma (distribución $\mathrm{t}=-2,403$; grados de libertad $\mathrm{gl}=131$; valor $\mathrm{p}=0,017$ ).

\section{Incidencia del establecimiento educacional de procedencia en el manejo del inglés del alumno}

Se analizaron las diferencias de resultados pre-test entre los distintos tipos de establecimientos educacionales de donde egresan de enseñanza media los estudiantes, ya sea Particular Pagado, Particular Subvencionado o Municipal, cuyo análisis de varianza no presenta diferencias estadísticamente significativas entre los tipos de colegio ( $F=1,12 ; p=0,327)$, lo que indica que la dependencia educacional de la que proceden los estudiantes no determina el manejo del inglés al ingresar a la Universidad (Figura 1).

Tras conocer que la dependencia educacional no afecta la variabilidad inicial de la muestra es que se analizaron las variables propias del proceso de enseñanza-aprendizaje.

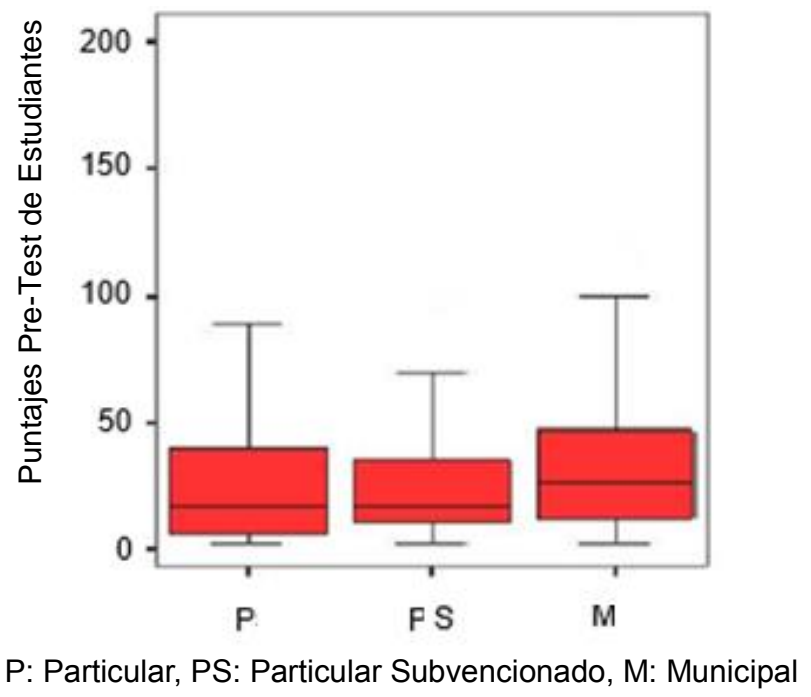

Fig. 1. Resultados del pre-test obtenidos mediante el tipo de establecimiento educacional de egreso 


\section{Influencia del profesor en el aprendizaje de los estudiantes}

A través de un análisis de varianza de un factor, se buscó conocer si el profesor afecta el avance en el aprendizaje del alumno. Los resultados muestran que existen diferencias estadísticamente significativas entre los profesores en función del progreso obtenido por los alumnos: prueba de igualdad de varianza $F=3,39$; grados de libertad $p=0,020$. Conocido esto, se hace necesario identificar que profesores marcan la diferencia en los resultados de los estudiantes, para lo cual se realizó una prueba Post-Hoc Tukey (Sheskin, 2003), que reveló la existencia de la única diferencia significativa observada entre ellos, donde el profesor $A$ obtendría mayores resultados que el profesor $\mathrm{D}(\bar{X}=16 ; p=0,14)$, expuesta en la Figura 2.

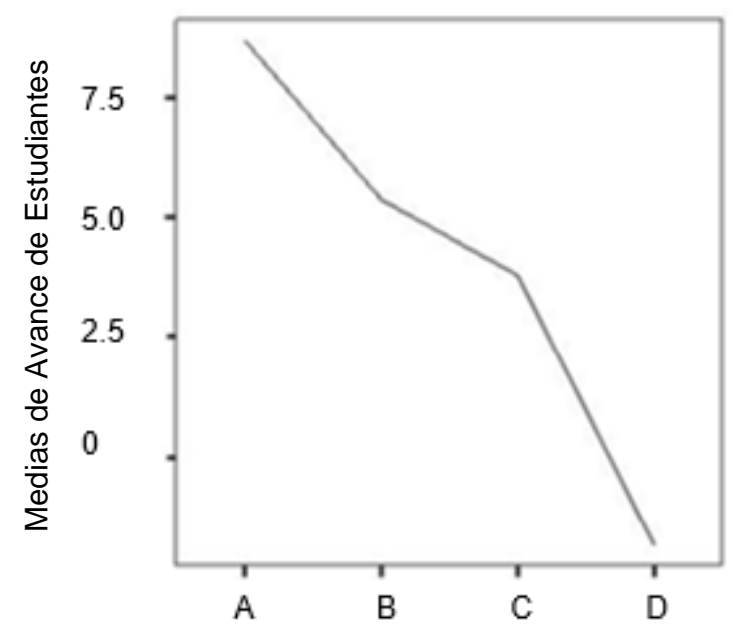

Fig. 2: Diferencias entre profesores A, B, C y D, en base a los resultados de los estudiantes.

\section{Diferencias entre Herramientas de apoyo}

Cuando se analiza el comportamiento que tienen las herramientas de apoyo en el proceso de aprendizaje de los estudiantes, no se logra identificar diferencias estadísticamente significativas entre ellas $(F=0,396 ; p=$ 0,674 ) aun cuando los contenidos fueron homologados y todos los estudiantes tenían un dominio similar del inglés al inicio del curso. Esto indica que ninguna de las herramientas se diferencia de otra en el efecto que causa en el aprendizaje del estudiante, sino que todas se comportan de manera similar. Sin embargo, se aprecia una leve tendencia que favorece al libro Top Notch, por sobre Interchange y DynEd (ver Figura 3). Por tanto, independiente de la herramienta que se utilice, los resultados obtenidos por los estudiantes serían los mismos, en contextos en que el rango de progreso en el aprendizaje es reducido.

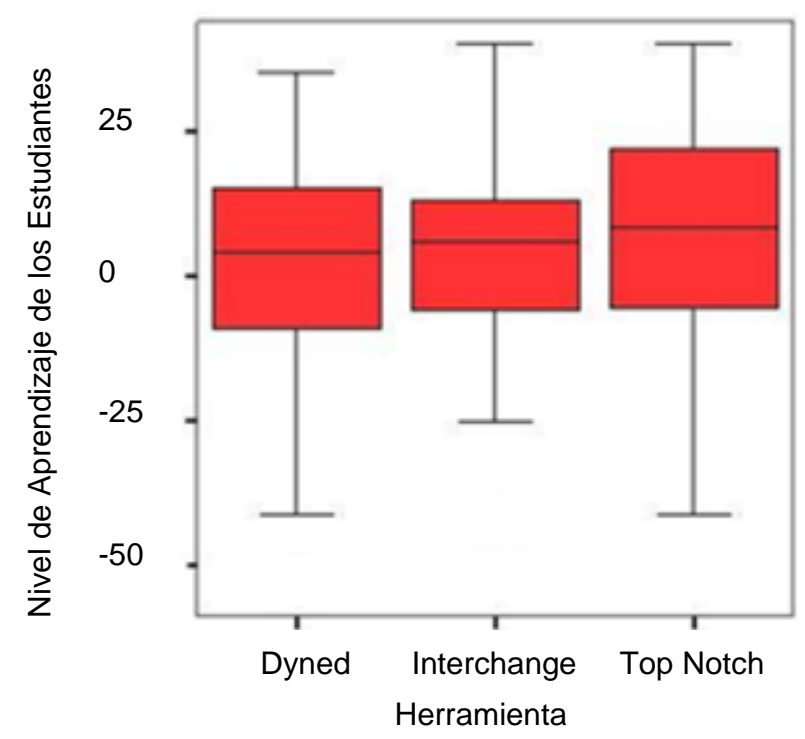

Fig. 3. Avances en aprendizaje de idioma Inglés según herramienta utilizada. 
Interacción entre el Profesor y Herramienta de apoyo

A fin de conocer si es el profesor como factor único el que genera diferencia estadísticamente significativa en el aprendizaje de los estudiantes, se decide realizar un análisis de interacción entre el profesor y la herramienta de apoyo, mediante análisis de varianza de dos factores. Los resultados indican que no existe interacción entre ambas vías, por lo tanto el avance no está determinado por el profesor - herramienta, sino que es el profesor por sí solo lo que genera un efecto estadísticamente significativo $(F=0,630 ; p=0,70)$ en el aprendizaje de los estudiantes. Por tanto, independiente de la herramienta de apoyo que se utilice, el resultado obtenido por los estudiantes en base al profesor, será el mismo.

\section{CONCLUSIONES}

De acuerdo a lo presentado y discutido en este trabajo, se obtienen las siguientes conclusiones principales:

1.- Si bien la literatura releva a las herramientas de apoyo como uno de los factores más influyentes en el aprendizaje del inglés, ninguna de estas supera a las otras en los resultados generales de los estudiantes, siendo el profesor el factor que más influye dentro del proceso. Un ejemplo de esto es que, a pesar de que se han creado sistemas para el aprendizaje de idiomas asistido por computador, que intentan emular el rol del profesor en la dinámica de enseñanza-aprendizaje, tal como presenta esta investigación, los resultados más bajos corresponden a los alumnos que fueron instruidos con herramienta de apoyo software, donde el rol del profesor se relega a un monitoreo de los datos entregados por la herramienta.

2.- En este estudio, se observó que dentro del nivel de colocación en el cual fueron ubicados los estudiantes, no existen diferencias en los resultados obtenidos de acuerdo al tipo de establecimiento de egreso. A diferencia de lo que la estadística en otras asignaturas reporta, en el aprendizaje del inglés, la dependencia educacional no predice el rendimiento que tengan los estudiantes.

3.- Los programas de inglés hoy en día, centran su foco en conseguir la mejor herramienta que garantice el aprendizaje de los alumnos. Con esta investigación, queda demostrado que la utilización de una u otra herramienta no determina directamente el rendimiento de los estudiantes. Por tanto, las perspectivas aquí presentadas representan una contribución teórica que promueve la re-estructuración y planificación de los programas de inglés en el ámbito universitario.

4.- Los resultados de este estudio apuntan a apoyar la importancia que tiene el idioma inglés como asignatura en la educación Chilena actual, ya que el aprendizaje del idioma Inglés es una necesidad para el desarrollo integral del alumno (Unidad de Currículum y evaluación, 2009). Claramente, ningún cambio será sustancial sin antes investigar los elementos relevantes del proceso, y monitorear constantemente la efectividad de este programa.

5.- Una línea de investigación, que emerge a la luz de este estudio, es el análisis de qué es lo que hace que un profesor sea más efectivo que otro, evaluando variables propias de este como lo son: la formación académica, el rol que cumple dentro de la clase y los estilos de enseñanza que utiliza.

\section{REFERENCIAS}

Al-Seghayer, $\mathrm{K}$, The effect of multimedia annotation modes on L2 vocabulary acquisition: a comparative study, Language Learning \& Technology, (5)1, 202-232 (2001)

Batista, J. y L. Salazar, Vigencia de los enfoques conductista, cognitivista y constructivista en la enseñanza del inglés, Revista Encuentro Educacional, 10 (3), 226-244, Universidad del Zulia, Venezuela (2003)

Blake, R., Review of computer-assisted foreign language teaching and learning technological advances, Language Learning \& Technology, 19 (1). $51-53$ USA (2015)

Brutt-Griffler, J., World englishes: a study of its developments, Multilingual Matters, USA (2002)

Carroli, P., Levels of Understanding of L2 Literary Texts Under Repeated Readings: Factors Contributing to Reading to Readers' Processing of Second Language Literature and Their Learning Outcomes, Conferencia Annual Meeting of the American Association of Applied Linguistics, St. Louis, USA, Febrero (2001)

Consejo de Europa, Marco Común Europeo de Referencia para las Lenguas: aprendizaje, enseñanza, evaluación, Ministerio de Educación, Cultura y Deporte/Instituto Cervantes, Madrid, España (2002) 
Constantinou, C. y Z. Zacharia, Computer Based Learning in Science, New Technologies and Their Applications in Education. 1, 845-851, (2003)

Derakhshan, A., R. Tamaskani y M. Faribi, Issues in language learning strategies. International Journal of Social Sciences and Education. 5(4), Turkía (2015)

Duran, A. y J. Barrios, Disposición y uso de recursos informáticos para la enseñanza-aprendizaje del inglés: una descripción a partir de una muestra en cien centros públicos de educación infantil y primaria de la Comunidad de Madrid, Portal Linguarum, 8, 193-223, España (2007)

Dyned, Dyned (en línea https://www.dyned.com/ acceso: 30 de Marzo de 2016), DynEd International (2014)

Emerging Tools, EmergingEdTools (en línea http://www.emergingedtech.com/2013/07/8-great-oonlineresources-for-esl-efl-teachers/ acceso 13 de Julio de 2016.

González, J., Integración de un modelo de proceso sistemático en el desarrollo de software educativo, Tesis de doctorado, Universidad de Vigo, España (2002)

Graham, C., Blended learning systems: Definition, current trends, and future directions. Handbook of blended learning: Global Perspectives, local designs, San Francisco: Pfeiffer Publishing, USA (2006)

Herramientas, 40 Herramientas TIC imprescindibles para el aula de inglés (en línea http://www.aulaplaneta.com/2015/09/17/recursos-tic/40-herramientas-tic-imprescindibles-para-el-aula-deingles/, acceso: 13 de Julio 2016.

Laufer, B., y M. Hill, What lexical information do L2 learners select in a CALL dictionary and how does it affect word retention? Language Learning \& Technology, (3) 2, 58-76, USA (2000)

Martínez, M., El nuevo papel del profesor universitario de lenguas extranjeras en el proceso de convergencia europea y su relación con la interacción, la tutoría y el aprendizaje autónomo, Porta linguarum, 7, 31-43, España (2007)

Moreno, F., La multimedia como herramienta para el aprendizaje autónomo del vocabulario del inglés por parte de los niños, Pedagogical Innovations, (3) 1, 84-94, Colombia (2011)

OET, Oxford English Testing (en línea

http://www.apcs.hcmus.edu.vn/LinkClick.aspx?fileticket=mll1w1DQDtY\%3D\&tabid=251\&language=en-US, acceso: 29 de Marzo de 2016), Oxford, UK (2016)

OPOT, Oxford Placement Online Test, (en línea

https://www.oxfordenglishtesting.com/DefaultMR.aspx?id=3034\&menuld=1, acceso: 29 de Marzo de 2016), Oxford University Press, Oxford, UK (2016)

Panteeva, K., Adapting stories for teaching English to young learners: A Brain-friendly learning perspective. International Journal of Education and Social Science, 2(6) UK, June (2015)

Pérez, J. M., S. Tejedor, J. F. Martínez y A. Julía, Tecnologia y pedagogía en las aulas: Perspectivas 2014El futuro inmediato en España, Gabinete de Comunicación y Educación de la Universidad Autónoma de Barcelona, España (2014)

Piquer, I., Aprender inglés en la escuela desde los tres años, Porta linguarum, 6, 115-128, España (2006)

Pressman, R., Ingeniería de software: un enfoque práctico, McGraw Hill, 7ma edición, España (2010)

Ramírez, D. y I. Alonzo, Using digital stories to improve listening comprehension with spanish young learners of English, Language Learning \& Technology, (11) 1, 87-101, Michigan State University, USA (2007)

Richards, J.; J. Hull; S. Proctor y C. Shields, Interchange, Cambridge University, 3era edición, Cambridge, UK (2005)

Saslow, J.; A. Ascher y B. Denman, Top Notch, Pearson Education, United States (2003) 
Sawaki, Y., Comparability of conventional and computerized tests of reading in a second language, Language Learning \& Technology, (5) 2, 38-59 (2001)

Segura, R., The importance of teaching listening and speaking skills. Trabajo fin de Master, Facultad de Educación, Universidad Complutense de Madrid, Madrid, España (2012)

Sheskin, D., Handbook of Parametric and Nonparametric Statistical Procedures, 3era edición, CRC Press (2003)

Silva, D.L., Innovación en la práctica docente. Revista Vinculando, Ciudad de México, México (2015)

Simasathiansophon, N., A perspective on blended-learning approach through course management system: Thailand's case study. International Journal of Information and Education Technology, (4) 2, Singapur, Abril (2014)

Subrahmanyam, M., M. Michikyan, C. Clemmon, R. Carrillo, Y. Uhis, y P.M. Greenfield, Learning from paper, learning from screens: Impact of screen reading and multitasking conditions on reading and writing among college students. International Journal of Cyber Behavior, Psychology ad Learning, 3(4), 1-27, Octubre (2013)

Thanasoulas, D., The changing winds and shifting sands of the history of English Language Teaching, English Club TEFL Articles (2002). https://www.englishclub.com/tefl-articles/history-english-languageteaching.htm Acceso: 26 de Marzo (2015)

Tuncer, U., How do monolingual and bilingual language learners differ in use of learning strategies while learning a foreign language? Evidences from Mersin University, Procedia - Social and Behavioral Sciences, (1) $1,852-856(2009)$

Unamuno, V., Lengua, escuela y diversidad sociocultural. Hacia una educación lingüística crítica, Grao, Barcelona (2003)

Unidad de Currículum y evaluación, Fundamentos del ajuste curricular en el sector de idioma extranjero, inglés, Santiago: Ministerio de Educación, República de Chile (2009)

Valdés, R., Puig, A., Aguirre, A., Reyes, E., Duarte, A., y Barata, A. Manual didáctico sobre la integración de las cuatro habilidades lingüísticas en la enseñanza del inglés, EDUMECENTRO, (4) 7, 56-70 (2015)

Vergara, M. y J. Alegría, Diversidad cultural: Un reto para las instituciones educativas, Universidad de Guadalajara, México. 1era Edición (2010)

Zou, B. y J.Li, Exploring mobile apps for English language teaching and learning, en F.Helm, L. Bradely, M.Guarda, y S. Thousëny (Eds), Critical CALL - Proceedings of the 2015 EUROCALL Conference, 564-568, Padova, Italia (2015) 
